\title{
Median PR Duration
}

National Cancer Institute

\section{Source}

National Cancer Institute. Median PR Duration. NCI Thesaurus. Code C102250.

A measurement of the median duration (time) of the PR interval, obtained from a set of measurements of the PR interval. The PR interval is defined as the time from the beginning of the $\mathrm{P}$ wave (representing the onset of atrial depolarization) to the beginning of the R wave (representing the onset of ventricular depolarization). In some cases, a Q wave will precede the R wave, in which case the PR interval is measured from the beginning of the $\mathrm{P}$ wave to the beginning of the $\mathrm{Q}$ wave. 